\title{
Production and characterization of attosecond electron bunch trains
}

\author{
Christopher M. S. Sears, ${ }^{*}$ Eric Colby, Rasmus Ischebeck, Christopher McGuinness, Janice Nelson, Robert Noble, \\ Robert H. Siemann, James Spencer, and Dieter Walz \\ Stanford Linear Accelerator Center, Menlo Park, California 94025, USA
}

Tomas Plettner and Robert L. Byer

Stanford University, Stanford, California 94305, USA

(Received 1 February 2008; published 5 June 2008)

\begin{abstract}
We report the production of optically spaced attosecond electron microbunches produced by the inverse free-electron-laser (IFEL) process. The IFEL is driven by a Ti:sapphire laser synchronized with the electron beam. The IFEL is followed by a magnetic chicane that converts the energy modulation into the longitudinal microbunch structure. The microbunch train is characterized by observing coherent optical transition radiation (COTR) at multiple harmonics of the bunching. Experimental results are compared with 1D analytic theory showing good agreement. Estimates of the bunching factors are given and correspond to a microbunch length of 410 attosec FWHM. The formation of stable attosecond electron pulse trains marks an important step towards direct laser acceleration.
\end{abstract}

DOI: 10.1103/PhysRevSTAB.11.061301

PACS numbers: 41.75.Jv, 41.60.Cr, 41.60.Dk, 41.85.Ct

\section{INTRODUCTION}

The continued development of short pulse, high peak intensity lasers has led to great interest in their application to particle acceleration. Recently, such lasers have succeeded in producing semimonoenergetic electron beams from plasma wakefield interactions [1-3]. These experiments relied on very large terawatt class systems and accelerated indirectly by inducing a "bubble"-shaped wake in a plasma and accelerating a beam of trapped plasma electrons. Lasers have also been used for direct acceleration between laser and electrons via inverse freeelectron-laser (IFEL) interactions [4,5], Inverse Cherenkov acceleration [6], and inverse transition radiation [7]. These experiments also relied on high power, low repetition rate lasers for acceleration. The transverse dimensions were much larger than the accelerating wavelength, leading to low coupling efficiency [8].

An altogether different approach to laser acceleration we are pursuing confines the electrons and laser in an optical scale accelerating structure. While the peak electric fields will still allow for a considerable accelerating gradient, approaching $1 \mathrm{GeV} / \mathrm{m}$ [9], the small dimensions mean the total pulse energy needed will be very low, just $\mu \mathrm{J} /$ pulse, and the coupling efficiency will be much greater. There exists a great deal of active research in optical scale structures, driven in large part by the telecommunications industry, including photonic band gap fibers [10] and lithographic optical components. Furthermore, while the laser system used in this experiment does not have impressive energy efficiency, there are great advances being made in

\footnotetext{
*cmsears@slac.stanford.edu
}

improving the efficiencies of ultrafast laser sources in the near-infrared [11].

To investigate near-field infrared laser acceleration, an IFEL microbuncher has been designed and tested at $800 \mathrm{~nm}$. Future net acceleration experiments will require longitudinally narrow bunches to achieve small energy spreads in downstream accelerators. Thus, examining the microbunches directly becomes important. IFEL production of microbunches has been demonstrated at $800 \mathrm{~nm}$ previously in a high-gain harmonic-generation (HGHG) experiment [12]. In that experiment, the study focused on the resulting radiation output, in large part ignoring characterization of the microbunches. Microbunches have also been produced and accelerated at $10.6 \mu \mathrm{m}$ [4]. The research reported in this paper marks an important first step in developing near-field IR laser accelerators where tolerances will be an order of magnitude tighter.

The microbuncher used in this experiment is comprised of a magnetic undulator in which the electron energy is modulated by an IFEL interaction and a dispersive chicane that converts the energy modulation into a density modulation. The IFEL alone has been demonstrated previously in an experiment exploring various harmonics of the IFEL interaction [13]. The IFEL interaction plus chicane produces a train of few hundred attosecond pulses spaced at the laser period (2.3 fs). Although microbunched electron beams have been well studied from FELs, producing them via an IFEL has the considerable added experimental difficulty of aligning the preexisting laser beam to the electrons. However, using an IFEL allows optical phase control of the microbunches, a requirement for net acceleration in a two-stage experiment. It also substantially reduces the length of the undulator required to form the microbunches. 


\section{MICROBUNCH FORMATION AND MEASUREMENT WITH COTR}

The interaction in the IFEL imparts a sinusoidal energy modulation along the electron beam, that is $\gamma=$ $\gamma_{0}+\eta \sin \left(k_{l} z_{0}\right)$, where $\eta$ is the amplitude of the modulation and $k_{l}$ is the laser wave number. Since the undulator in this experiment is very short, the change in a particle's position within the beam during the IFEL interaction is negligible. Following the undulator, the chicane imparts an energy dependent path length change $d z=R_{56}(\gamma-$ $\left.\gamma_{0}\right) / \gamma_{0}$, where $R_{56}$ is the temporal dispersion of the chicane. The $R_{56}$ of the chicane is found by integrating the Lorentz equations and is given by

$$
R_{56}=\frac{L}{\gamma^{2}}+\left(\frac{q}{\gamma m_{e} c}\right)^{2} \int_{0}^{L}\left[\int_{-\infty}^{z} B_{y}\left(z^{\prime}\right) d z^{\prime}\right]^{2} d z .
$$

Here, $L$ is the distance from the undulator to the radiator where the microbunches are measured and $B_{y}$ is the chicane magnetic field. The first term in the expression comes from velocity bunching which gives a small but nonnegligible contribution to the total $R_{56}$ (about 5\%). The chicane dispersion skews the longitudinal phase space forming a density modulation with strong harmonic content. This density modulation $\rho(z)$ can be expressed as a Fourier series whose coefficients are Bessel functions depending on the amount of dispersion $\left(R_{56}\right)$ and the modulation from the IFEL $[14,15]$ by

$$
\rho(z)=\rho_{0}\left[1+2 \sum_{n=1}^{\infty} b_{n} \cos \left(n k_{l} z\right)\right]
$$

where

$$
b_{n}=J_{n}\left(n k_{l} R_{56} \frac{\eta}{\gamma_{0}}\right) \exp \left[-\frac{n^{2}}{2}\left(k_{l} R_{56} \frac{\sigma_{\gamma}}{\gamma_{0}}\right)^{2}\right],
$$

$\rho_{0}$ is the initial charge density entering the IFEL, and $\sigma_{\gamma}$ is the initial electron energy spread. The exponential term in the expansion coefficients defines the washout of the bunching due to the initial energy spread. The bunching is optimized for $k_{l} R_{56} \eta / \gamma_{0}=1-1.5$ depending on the harmonic number $n$. For fixed $k_{l} R_{56} \eta / \gamma_{0}=1$, the exponential term can be written as $\exp \left[-0.5 n^{2}\left(\sigma_{\gamma} / \eta\right)^{2}\right]$. Thus, when $\sigma_{\gamma}>\eta$ the washout due to the energy spread becomes significant. Other effects can cause microbunch washout including electron beam divergence [14] and space charge [16]. However, in this experiment these effects are not significant due to the relatively mild focusing $\left(\sigma_{\theta} \simeq 2 \times 10^{-4}\right.$ radians $)$ and low charge $(\sim 1 \mathrm{pC})$.

To verify the existence of the microbunches, the electron beam is sent through a foil to produce coherent optical transition radiation (COTR). The total intensity of transition radiation is given by $[17,18]$

$$
\frac{d I_{\text {tot }}}{d \Omega d \omega}=N\left[1+(N-1)|f|^{2}\right] \frac{d I_{\text {sing }}}{d \Omega d \omega},
$$

where

$$
f=\frac{1}{Q} \int \rho(\vec{x}) \exp (i \vec{k} \vec{x}) d \vec{x} .
$$

$I_{\text {sing }}$ is the transition radiation due to a single electron [19] and $f$ is the structure function equal to the Fourier transform of the charge distribution. The transform of the longitudinal density modulation [Eq. (2)] produces a set of frequencies at harmonics of the laser used to bunch the electron beam and the structure function becomes

$$
f=\delta(\omega)+2 \sum_{n=1}^{\infty} b_{n} \delta\left(\omega-n k_{l} c\right) .
$$

Equation (6) can be further refined by including in the bunch density the longitudinal beam envelope of $\sigma_{z} \sim$ $0.3 \mathrm{~mm}$. The longitudinal envelope changes the $\delta$-functions to Gaussians with a bandwidth given by $\lambda_{0}^{2} /\left(2 \pi \sigma_{z}\right) \simeq 0.3 \mathrm{~nm}$, much narrower than the spectral acceptance of the detectors used in the experiment.

Over the range of frequencies of interest, the transition radiation of a single particle has no frequency dependence. Thus, the only frequency dependence in the coherent transition radiation is due to the microbunching itself. In this way, measuring the COTR is a direct measurement of the microbunch structure. That is, the total radiated energy at each harmonic is proportional to the square of the bunching coefficients:

$$
I_{\mathrm{COTR}, n} \propto\left[J_{n}\left(n k_{l} R_{56} \eta\right)\right]^{2} \exp \left[-\left(\frac{n k_{l} R_{56} \sigma_{\gamma}}{\gamma_{0}}\right)^{2}\right] .
$$

In characterizing the microbunches, the goal of the experiment is to verify the applicability of Eq. (7) through the dependence of COTR yield on the chicane strength, the $R_{56}$, as well as the modulation strength $\eta$. Direct comparison of the experimental results to the analytic theory is complicated by several issues, most appreciably the nonuniformity of the laser field, both transversely and in time. In the real experiment there is not a constant modulation $\eta$ for every electron in the bunch. As a result, certain portions of the electron beam will optimally bunch at different values of $R_{56}$ and will similarly radiate most strongly at different values. Nevertheless, we expect the averaged COTR yield to maintain the functional dependence shown in Eq. (7).

\section{THE EXPERIMENT}

This research was conducted at the NLCTA facility at SLAC, a $60 \mathrm{MeV}$ X-band accelerator that was recently upgraded with an S-band photoinjector for laser acceleration research. The NLCTA beam line includes a chicane for energy collimation. Using the energy collimation, the accelerator produces $1 \mathrm{pC}, \sim 1 \mathrm{ps}$ pulses with $\leq 0.05 \%$ energy spread. The laser system used to produce UV for the photoinjector also provides IR pulses for laser accel- 
TABLE I. Experimental parameters for attosecond bunch train production. All widths are given as FWHM.

\begin{tabular}{lc}
\hline \hline Parameter & Value \\
\hline Electron energy & $60 \mathrm{MeV}$ \\
Electron energy spread & $30 \mathrm{keV}$ (typical) \\
Electron energy jitter & $6 \mathrm{keV}$ \\
Electron pulse length & $0.8 \mathrm{ps}^{\mathrm{a}}$ (typical) \\
Electron timing jitter & $<0.2 \mathrm{ps}^{\mathrm{a}}$ \\
Electron spot size & $100 \mu \mathrm{m}(\mathrm{nominal})$ \\
Electron transverse jitter $(x$ and $y)$ & $25 \mu \mathrm{m}$ \\
Bunch charge & $1 \mathrm{pC} \mathrm{(nominal)}$ \\
Laser wavelength & $785 \mathrm{~nm}$ \\
Laser energy & $0.65 \mathrm{~mJ} / \mathrm{pulse}$ \\
Laser pulse length & $0.55 \mathrm{ps}$ \\
Laser spot size & $200 \mu \mathrm{m}$ \\
Undulator period & $1.8 \mathrm{~cm}$ \\
Number of periods & 3 \\
Undulator strength $\left(a_{w}\right)$ & 0.46 \\
Chicane $R_{56}$ & $0.04-0.16 \mathrm{~mm}$ \\
\hline \hline
\end{tabular}

${ }^{\mathrm{a}}$ Estimated from the laser interaction data.

eration research. This setup gives the timing stability necessary for the experiments [20].

Table I gives a list of experimental parameters including electron, laser, and magnet values. The electrons enter a separate experimental hall via a $25^{\circ}$ dogleg bend. The laser for the IFEL is introduced at an optical window at the second dogleg bend. Both beams are focused going into the experimental chamber housing the undulator, chicane, and the COTR radiator as well as several diagnostic screens (see Fig. 1). Two Ce:YAG screens are attached fore and aft of the undulator to aid in overlapping the laser and electron beam, while a third screen is used to minimize the electron beam spot size at the radiator. A Cherenkov radiator is also located in the experimental chamber for obtaining picosecond-class timing of the laser to electron beam using a streak camera. After the experimental chamber the electrons enter a $90^{\circ}$ imaging energy spectrometer with $5 \mathrm{keV}$ resolution. The COTR radiator is located at the object point of the energy spectrometer coinciding with the minimum electron spot size. This gives optimal resolution at the spectrometer and prevents the induced scatter from the foil from affecting the measured energy spectrum. To avoid damaging the COTR radiator and to reduce background due to the laser, a tungsten beamstop is inserted in the middle of the chicane to intercept the laser after it is used for the IFEL.

The radiator consists of two pellicle mirrors separated by $15 \mathrm{~mm}$, one normal to the electron beam and a second at $45^{\circ}$ to send the light to a pair of photomultiplier tubes (PMTs). Thus, the radiator actually produces two bursts of COTR, one from each pellicle surface. The light collecting optics spatially and spectrally integrate any interference pattern (acceptance angle $\sim 100 \mathrm{mrad}$ and spectral

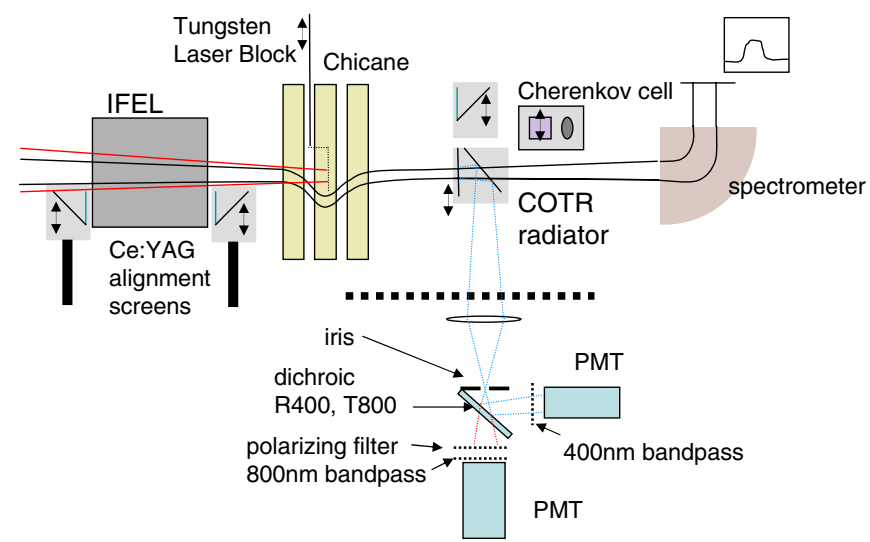

FIG. 1. (Color) Layout of the microbunching experiment. An IFEL and chicane form microbunches that then pass through a pellicle mirror. The beam emits transition radiation at harmonics of the IFEL drive laser. The transition radiation is collected and split to two PMT detectors observing the first and second harmonics of the bunching. An electron energy spectrometer is used for monitoring the strength of the IFEL interaction and correlating to transition radiation intensity.

bandwidth $=40 \mathrm{~nm}$ ). A lens images the first pellicle onto an iris which is closed down to limit the field of view to only that pellicle. This helps reduce background signal on the $800 \mathrm{~nm}$ PMT due to scattered light from the laser in the experiment chamber. The COTR light is then split using a dichroic mirror to reflect the second harmonic to one PMT and transmit the fundamental to the second. Each PMT also has a bandpass filter (bandwidth of $40 \mathrm{~nm}$ ) attached to select only the given harmonic and also help protect against ambient light. Finally, a polarizing filter is placed in front of the $800 \mathrm{~nm}$ PMT to further reduce background due to the laser.

Data are taken in sets of 500-4000 electron-laser interaction events at $10 \mathrm{~Hz}$. A fast, movable retroreflector is used to vary the delay between the electron beam and laser before each event. For each event an image of the electron beam at the energy spectrometer is acquired along with numerous diagnostics including the COTR PMTs and diodes for the laser. From the energy profiles, the energy spread is extracted and plotted against the delay for each event forming a cross-correlation scatter plot (Fig. 2). When the electron beam and laser are in time the IFEL modulates the electron beam energy and the COTR signal is strongest.

The cross correlation is an important technique for this experiment, especially for the COTR signal. The fact that the signals from the PMTs are strongest when the laser and electron beam are in-time excludes other processes as background including harmonic generation from the laser hitting surfaces in the chamber, incoherent transition radiation, bremsstrahlung, or undulator radiation, all of which would occur regardless of relative timing between the two beams. 

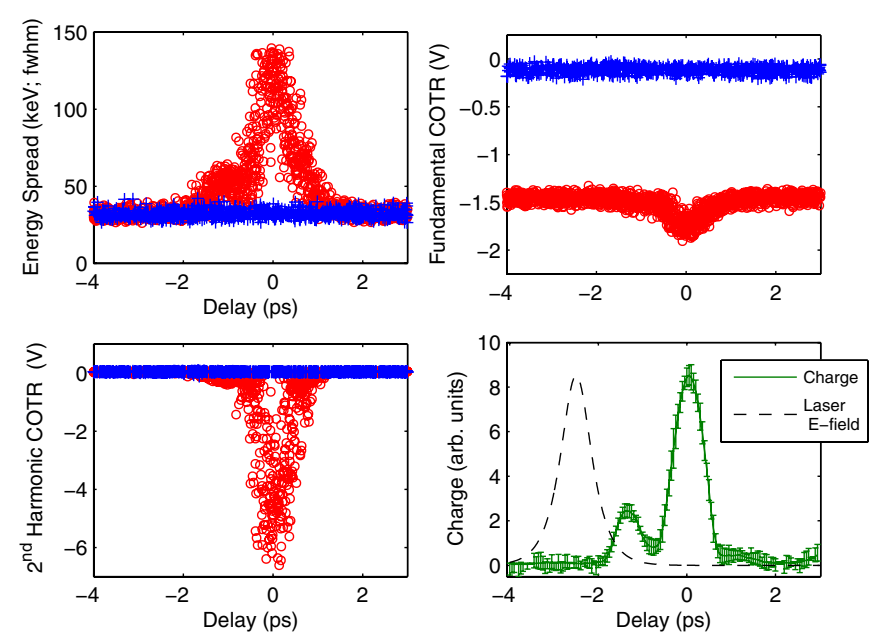

FIG. 2. (Color) Example data run of the IFEL microbunching experiment containing 2130 events. Top left is the electron energy spread as a function of delay with laser-on events in red (circles), laser-off in blue (crosses). Also shown are the COTR raw signals at the fundamental (top right) and second harmonic (bottom left). The bottom right figure shows the current profile calculated by deconvolving the laser pulse from the cross-correlation data.

\section{RESULTS}

Figure 2 depicts an example data set showing the crosscorrelation scatter plots of the energy spread as well as the fundamental and second harmonic COTR signals. Notice that when the delay is set for laser-electron temporal overlap, all events show interaction. This indicates that the timing jitter is less than the electron and laser pulse widths. The temporal width of the slopes on the cross correlation give an upper bound on the jitter of $0.2 \mathrm{ps}$. In this particular electron beam configuration there is a smaller electron pulse behind the main pulse. The COTR signals show the same characteristic structure to the electron beam. The fundamental COTR signal has a large offset between laser-on and laser-off events indicating there is still significant bleed-through of laser light to the detector. This bleedthrough also contributes significantly to the noise on the signal.

Deconvolution of the laser temporal profile from the electron energy modulation (Fig. 2, bottom right) estimates the main electron pulse temporal length of 0.8 ps FWHM. The laser temporal profile, obtained from autocorrelation, is $0.55 \mathrm{ps}$. The IFEL modulation is given by the difference in quadrature of the total energy spread and the initial energy spread $\left(\sigma_{\mathrm{IFEL}}=\sqrt{\sigma_{\mathrm{TOT}}^{2}-\sigma_{\text {init }}^{2}}\right)$. The energy modulation cross correlation is binned as a function of delay and deconvolved from the laser field profile using a genetic algorithm [21]. The deconvolution is performed several times, reseeding with an energy modulation spectrum that is varied using the variances of the binned data.

Figure 3 shows a plot of COTR output versus the IFEL modulation strength. Here, the raw COTR signals have
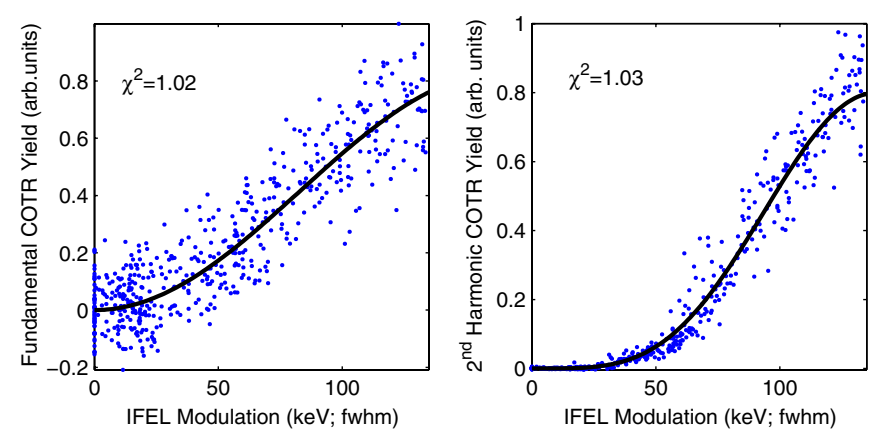

FIG. 3. (Color) Scatter plot of COTR signals versus IFEL modulation for a single run. Each point is a single interaction of the laser and electron beam. The amplitude of the IFEL interaction varies due to the delay scan. The solid lines give the analytic form [Eq. (7)].

been inverted, rescaled, and the baseline signal (with no IFEL interaction) subtracted. The only cut applied to the data is on the delay timing to select events near the interaction overlap.

Also shown in Fig. 3 together with the data for each harmonic is the analytic form [Eq. (7)] using the known value of $R_{56}=0.13 \mathrm{~mm}$ and taking $\eta=0.5 \cdot M \cdot 1.3$, where $M$ is the measured IFEL modulation FWHM. For a constant modulation across the entire electron beam, we would have $\eta=0.5 \cdot M$ (recall $\eta$ is the amplitude of a sinusoidal modulation). However, since the measured $M$ is an average across the entire electron beam including electrons not modulated (either out-of-time or off-axis transversely), we expect the radiating portion of the electron beam to have a larger modulation $\eta$. Allowing $\eta$ as a fit parameter and minimizing $\chi^{2}$ we obtain $\eta=0.65 \cdot M$. The fit of the analytic form to the data shows very good agreement with $\chi^{2}=1.02$ for the fundamental data and $\chi^{2}=1.03$ for the second harmonic. For this fit the variance for each data point is calculated from the deviation of the nearest neighbors in IFEL modulation. With the COTR signals plotted in this way the difference in the two harmonics is clear. The second harmonic does not begin to appear strongly until much larger values of the IFEL modulation. This can be directly related to the differing Bessel function dependence in Eq. (7).

In addition to the modulation strength, $\eta$, the COTR output varies strongly with the chicane $R_{56}$ (Fig. 4). The chicane design includes coils to give adjustment of the magnetic field by up to $\pm 20 \%$ in order to optimize microbunch formation. To study the dependence of COTR output versus chicane strength, a number of small data sets were taken with the chicane strength varied between each set. A fit was done to each cross correlation, an amplitude extracted, and an error deduced using the boot-strap method [22].

The main difference between the two curves in the chicane scan comes at low values of $R_{56}$ where the second harmonic again rolls off faster than the fundamental. At 


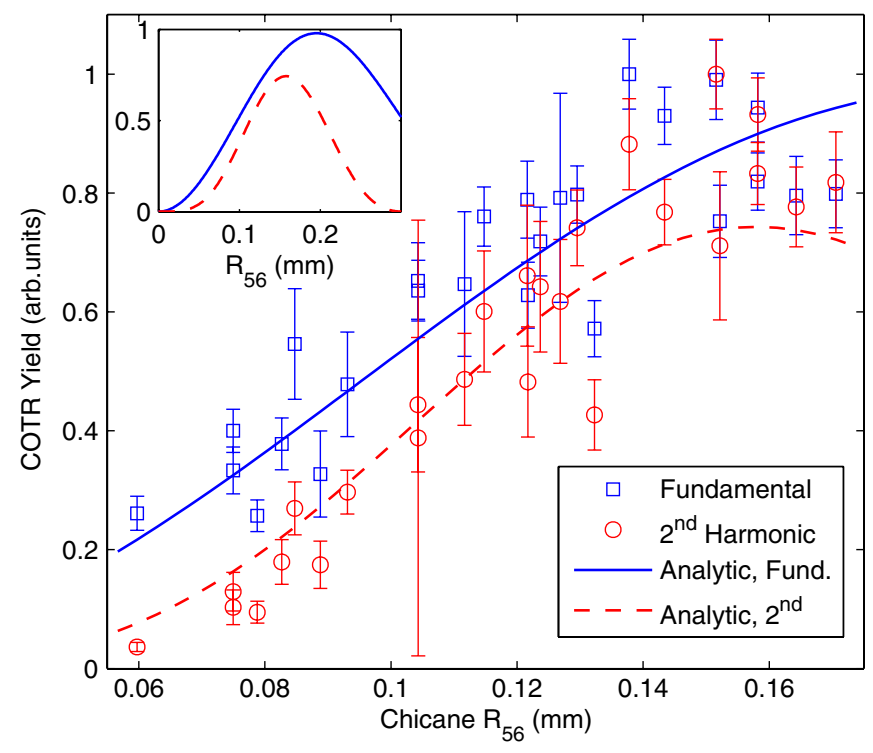

FIG. 4. (Color) A scan of the chicane $R_{56}$. The first and second harmonics are shown together with the analytic form overlayed. Each data point represents a run of 500 events, fitted with errors found by the boot-strap technique. The inset shows the analytic function for the COTR output [Eq. (7)].

higher values both signals show a roll-off indicating that optimum bunching is being reached for $R_{56}=$ $0.13-0.16 \mathrm{~mm}$. The inset shows the analytic form over a larger range of $R_{56}$ taking $\eta=0.65 \cdot M$ as before where for these runs the mean interaction $M=100 \mathrm{keV}$ FWHM. These runs were taken over a period of $\sim 1$ hour during which both the mean interaction strength $M$ and the total charge drifted. As a result, the fit to the analytic form is relatively poor compared to that of the COTR versus modulation data shown in Fig. 3. Nevertheless, there is qualitative agreement with the analytic form. The analytic solutions reach a maximum at $R_{56}=0.20 \mathrm{~mm}$ for the fundamental and $0.16 \mathrm{~mm}$ for the second harmonic. The actual COTR signals peak for lower $R_{56}$ due to the mean being pulled down by more strongly interacting events that are overbunched. Figure 2 shows events with modulation up to $140 \mathrm{keV}$. For these events, optimal bunching would occur at $R_{56}=0.12 \mathrm{~mm}$. This demonstrates the importance of independently measuring the bunching through the COTR to optimize the $R_{56}$.

A number often quoted as a figure of merit for bunching is the bunch parameter $b_{n}$, equal to the Fourier coefficient for longitudinal charge density. Recall that $b_{n}=$ $J_{n}\left(n R_{56} k_{L} \eta / \gamma_{0}\right) \exp \left[-\frac{1}{2}\left(n k_{l} R_{56} \sigma_{\gamma} / \gamma_{0}\right)^{2}\right]$. Given the good agreement between the analytic theory and the data demonstrated in the energy modulation and chicane strength scan data, we can use the analytic theory to calculate the bunching parameters and resulting microbunch density given by Eq. (2). For the measured values of $R_{56}=0.16 \mathrm{~mm}$ and the modulation $M=140 \mathrm{keV}$ we infer, using Eq. (3), that $b_{1}=0.52$ and $b_{2}=0.36$. Figure 5

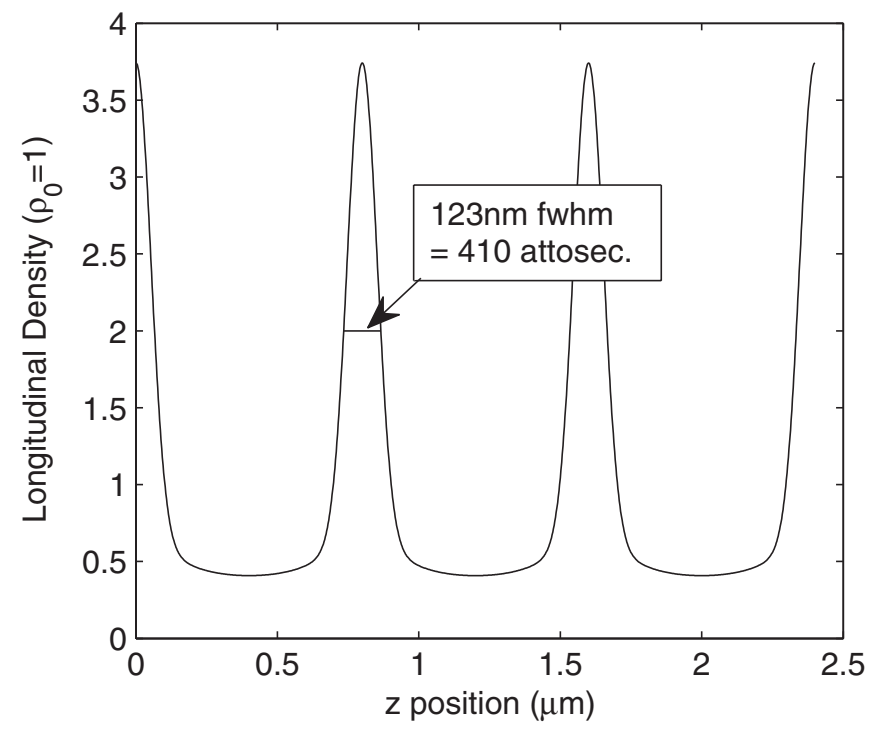

FIG. 5. Longitudinal electron density versus position [Eq. (2)] at the center of the electron bunch using the measured experimental values: $R_{56}=0.16 \mathrm{~mm}, \eta=0.5 \cdot 140 \mathrm{keV} / 60 \mathrm{MeV}$, and $\sigma_{\gamma}=17 \mathrm{keV}$ (rms). At the edges of the bunch, both longitudinally and transversely the laser field is weaker and corresponding microbunch formation weaker.

shows the longitudinal electron density versus position near the center of the bunch as predicted from Eq. (2) using the experimental parameters. At this level of bunching the individual microbunches have a temporal pulse width of $\sim 410$ attosec FWHM and the peak charge is larger by a factor of 3 from the unbunched charge density. Of course, since the modulation strength varies over the bunch due to the roll-off of the laser field intensity, the microbunch density will decrease towards the edges of the bunch.

\section{CONCLUSION}

This experiment has succeeded in producing stable, optically spaced attosecond bunch trains. The microbunches are characterized through coherent optical transition radiation (COTR), and show good agreement with an analytic theory for microbunch formation. With the laser to electron beam delay held constant, a microbunched beam is produced on every shot with a jitter corresponding to $\leq$ $20 \%$ on the bunching factor. This stability will be important in future experiments with the goal of achieving acceleration microbunches in near-field optical structures such as photonic crystal fibers [10]. Prior to laser powered acceleration experiments, the microbunched beam will also be used to probe candidate accelerator structures by studying the wakes produced by the passage of a microbunched beam through a near-field optical structure [23].

The production of a microbunched beam may also find useful applications outside of direct laser acceleration. As already noted in the Introduction, microbunched beams have already been produced for HGHG FELs, and further 
experimentation in this direction is planned by researchers around the world [24]. Microbunching may also be useful in driving plasma wakes [25] or as a Compton scattering source.

This research effort might one day lead to novel devices producing energetic beams at very high repetition rates. With the application of lithographic processing techniques and commercially mass produced laser components these devices could be made cheap, compact, and reliable.

\section{ACKNOWLEDGMENTS}

The authors wish to acknowledge the efforts and contributions of the NLCTA Operations group: Justin May, Doug McCormick, Tonee Smith, Richard Swent, and Keith Jobe. We would also like to thank Walt Zacherl and Bruce Rohrbough for their work on laser diagnostics. This work is supported by Department of Energy Contracts No. DEAC02-76SF00515 and No. DE-FG02-03ER41276.

[1] J. Faure, Y. Glinec, A. Pukhov, S. Kiselev, S. Gordienko, E. Lefebvre, J. Rousseau, F. Bourgy, and V. Malka, Nature (London) 431, 541 (2004).

[2] S. P. D. Mangles et al., Nature (London) 431, 535 (2004).

[3] C. Geddes, C. Toth, J. van Tilborg, E. Esarey, C. Schroeder, D. Bruhwiler, C. Nieter, J. Cary, and W. Leemans, Nature (London) 431, 538 (2004).

[4] W. D. Kimura et al., Phys. Rev. Lett. 86, 4041 (2001).

[5] P. Musumeci et al., Phys. Rev. Lett. 94, 154801 (2005).

[6] W. D. Kimura, G. H. Kim, R. D. Romea, L. C. Steinhauer, I. V. Pogorelsky, K. P. Kusche, R. C. Fernow, X. Wang, and Y. Liu, Phys. Rev. Lett. 74, 546 (1995).

[7] T. Plettner, R. L. Byer, E. Colby, B. Cowan, C. M. S. Sears, J.E. Spencer, and R. H. Siemann, Phys. Rev. Lett. 95, 134801 (2005).

[8] Y. C. N. Na, R. H. Siemann, and R. L. Byer, Phys. Rev. ST Accel. Beams 8, 031301 (2005).
[9] B. M. Cowan, Phys. Rev. ST Accel. Beams 6, 101301 (2003).

[10] X. E. Lin, Phys. Rev. ST Accel. Beams 4, 051301 (2001).

[11] P. Klopp, V. Petrov, U. Griebner, K. Petermann, V. Peters, and G. Erbert, Opt. Lett. 29, 391 (2004).

[12] L. H. Yu et al., Phys. Rev. Lett. 91, 074801 (2003).

[13] C. M. S. Sears, E. R. Colby, B. M. Cowan, R. H. Siemann, J.E. Spencer, R. L. Byer, and T. Plettner, Phys. Rev. Lett. 95, 194801 (2005).

[14] S. Baccaro, F. D. Martini, and A. Ghigo, Opt. Lett. 7, 174 (1982).

[15] A. Luccio, G. Matone, L. Miceli, and G. Giordano, Laser Part. Beams 8, 383 (1990).

[16] L. C. Steinhauer and W. D. Kimura, Phys. Rev. ST Accel. Beams 2, 081301 (1999).

[17] J. S. Nodvick and D. S. Saxon, Phys. Rev. 96, 180 (1954).

[18] Y. Shibata, T. Takahashi, T. Kanai, K. Ishi, M. Ikezawa, J. Ohkuma, S. Okuda, and T. Okada, Phys. Rev. E 50, 1479 (1994).

[19] V. L. Ginsburg and I. M. Frank, JETP 16, 15 (1946).

[20] C. McGuinness, R. Byer, E. Colby, R. Ischebeck, R. Noble, T. Plettner, C. M.S. Sears, R. Siemann, J. Spencer, and D. Walz, in Proceedings of PAC 2007, Albuquerque, New Mexico, USA (IEEE, Piscataway, NJ, 2007), p. 4195.

[21] M. Mitchell, An Introduction to Genetic Algorithms (Complex Adaptive Systems) (The MIT Press, Cambridge, MA, 1998).

[22] M.R. Chernick, Bootstrap Methods, A Practitioner's Guide, Wiley Series in Probability and Statistics (WileyInterscience, New York, 1999).

[23] C. M.S. Sears et al., in Proceedings of PAC 2007, Albuquerque, New Mexico, USA, Ref. [20], p. 3106.

[24] D. Krämer, E. Jaeschke, and W. Eberhardt, The BESSY Soft X-ray Free Electron Laser (BESSY, Berlin, 2004), ISBN 3-9809534-08.

[25] E. Kallos, T. Katsouleas, W. D. Kimura, K. Kusche, P. Muggli, I. Pavlishin, I. Pogorelsky, D. Stolyarov, and V. Yakimenko, Phys. Rev. Lett. 100, 074802 (2008). 